\title{
An exceptionally large sarcoma of the thoraco-abdominal wall with ingrowth in the pericardium and stomach: a case report
}

\author{
Alexander P. W. M. Maat ${ }^{1}$, Mischa M. Zuijdendorp ${ }^{2}$, Geert J. L. van Leenders ${ }^{3}$, Alexander Hirsch ${ }^{4}$, \\ Joost Rothbarth ${ }^{5}$ \\ ${ }^{1}$ Department of Cardiothoracic Surgery, ${ }^{2}$ Department of Plastic and Reconstructive Surgery, ${ }^{3}$ Department of Pathology, ${ }^{4}$ Department of Cardiology \\ and Radiology and Nuclear Medicine, ${ }^{5}$ Department of Oncological and Gastro-intestinal Surgery at Erasmus MC, University Medical Center \\ Rotterdam, Rotterdam, The Netherlands \\ Correspondence to: Alexander P. W. M. Maat, MD. Department of Cardiothoracic Surgery, Thorax Center, Erasmus MC, University Medical Center \\ Rotterdam, Doctor Molewaterplein 40, Room Rg 629, PO Box 2040, 3015 GD Rotterdam, The Netherlands. Email: a.p.w.m.maat@erasmusmc.nl.
}

\begin{abstract}
A 62-year-old man was referred to our center with a sarcoma of his left chest wall with ingrowth into the pericardium and transdiaphragmatically into the abdomen. The diagnostic delay for patient was extensive, at approximately 3.5 years. Judging by the tumor size and local ingrowth, on first examination, we considered this tumor to be borderline resectable. The patient was treated with induction radiotherapy, resulting in a relief of pain but no radiological response. He was then re-evaluated with particular attention paid to the (peri)cardial involvement. Magnetic resonance imaging (MRI) showed the heart could move freely, and therefore extended resection and reconstruction of the chest and abdominal wall, pericardium, and diaphragm was performed. The combination of diagnostic delay, the large tumor size, the extensive resection, and the use of a nonrigid material for the reconstruction make this case unique. A computed tomography (CT) scan performed 6 weeks postoperatively showed signs of abdominal and lung metastases, despite a "clean" CT scan taken a few days preoperatively. The main lessons from this case are that a slow, progressive, painful swelling of the chest wall should never be diagnosed as a chronic contusion and that a very large thoraco-abdominal wall defect can be reconstructed without the use of rigid materials.
\end{abstract}

Keywords: Thoraco-abdominal wall resection; pericardium; diaphragm; patient and doctor delay; case report

Received: 31 March 2020; Accepted: 10 July 2020; Published: 25 May 2021.

doi: $10.21037 /$ ccts-20-93

View this article at: http://dx.doi.org/10.21037/ccts-20-93

\section{Introduction}

Sarcomas are rare primary tumors of the thoracic wall, with chondrosarcoma being the most frequently encountered type. In The Netherlands, the incidence rate for chondrosarcoma is 8.78 per million (1), with the incidence being highest in patients between 50 and 70 years of age. Persistent pain and a growing mass are the two main presenting complaints. Chondrosarcoma's are known to be chemotherapy and radiation resistant, and so a radical resection is the only valid curative option. Grade 3 dedifferentiated chondrosarcoma's carry a dismal prognosis due to their tendency for distant metastases (2). It is estimated that in about $10 \%$ of cases, a low-grade chondrosarcoma will dedifferentiate into a malignant grade 3 type given time (3-5). Although the exact molecular pathogenetic sequence of dedifferentiation in a chondrosarcoma is unknown, aberrations in cellcycle regulators $\mathrm{p} 16, \mathrm{p} 53$, and $\mathrm{Rb}$ seem to play a role in this process (6). We present this case in accordance with the CARE reporting checklist (available at https://ccts. amegroups.com/article/view/10.21037/ccts-20-93/rc).

\section{Case presentation}

A 62-year-old man developed a slowly progressive painful mass in his left antero-lateral chest wall, which was first noticed in 2016. He lived an active life as a painter, running his own one-man business and actively engaging in regular 

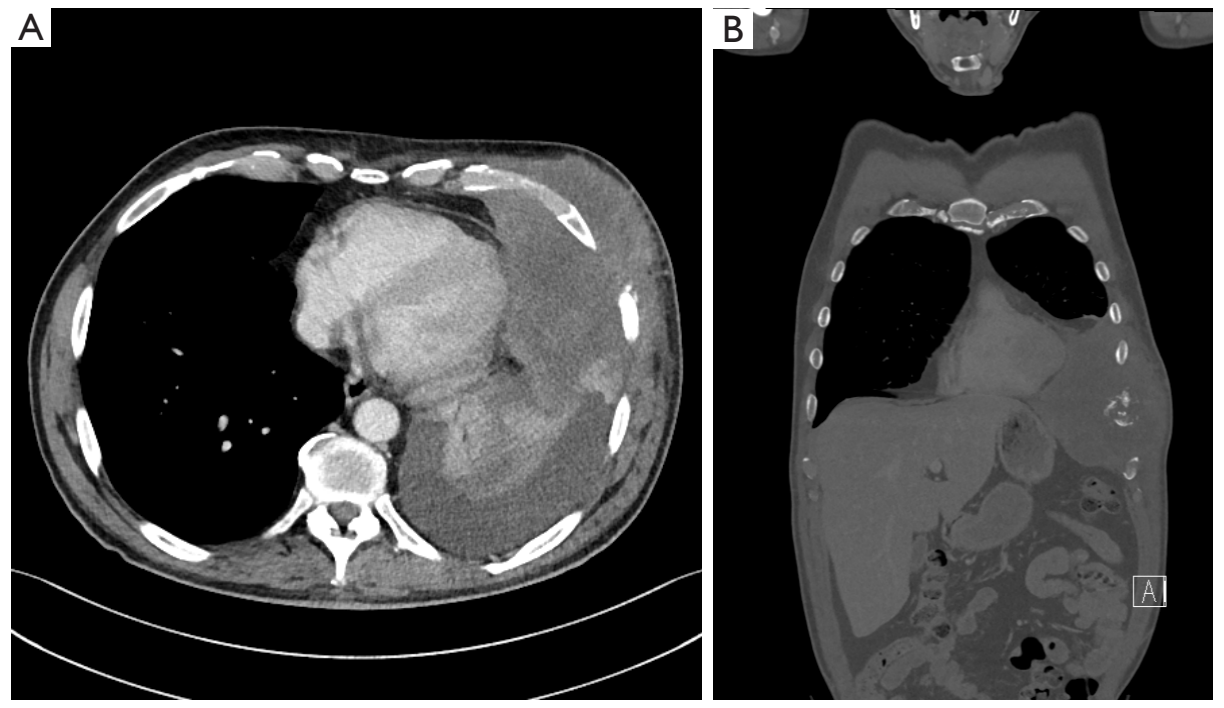

Figure 1 Sagital and coronal view of the tumor (CT scan November 19, 2019)—compression on the heart and rib destruction are clearly visible.

sports activities. Over a period of 20 years, he was involved in 3 motorcycling accidents, but did not sustain any injuries. He consulted his general practitioner (GP) at least 5 times between 2017 and 2018 for a gradually increasing pain and a swelling of his left chest. The GP's diagnosis was chronic contusion, and the patient was advised to use more pain medication.

In January 2019, the GP was consulted again because of increasing pain. A large lump was visible and palpable, and a chest X-ray revealed no abnormalities. No further investigations were performed at this point, as the working diagnosis was still deemed to be chronic contusion despite the lack of recent trauma or any hematoma in the area. The patient was eventually referred to a pain management team; however, he was never evaluated by this team due to a long waiting list.

A few months later, the patient was forced to stop working due to the persistent and debilitating pain. He had a pain score of $6 / 10$ points (persisting into the night) on the visual analogue scale and was on multidrug pain medication (paracetamol $4 \times 1 \mathrm{~g}$ daily, naproxen $4 \times 250 \mathrm{mg}$. daily, and oxycodone up to $4 \times 5 \mathrm{mg}$ daily).

In May 2019, he consulted another GP who suspected a serious problem; an ultrasound revealed a large solid tumor of the chest wall. Subsequently, the patient was referred to a local hospital: a computed tomography (CT) scan showed a large tumor of the chest wall $(16 \mathrm{~cm} \times 12 \mathrm{~cm} \times 12 \mathrm{~cm})$ with encasement of ribs $6-8$, attached to the pericardium and growing through the diaphragm into the abdomen, without signs of distant metastases (Figure 1). Puncture revealed atypical spindle cells, but the pathologist was not able to make a definite diagnosis. A second opinion performed by our soft-tissue pathologist revealed a low-grade sarcoma of the chest wall.

Subsequently, the patient was referred to the multidisciplinary sarcoma team in our hospital. The tumor was considered borderline resectable, and our tumor board decided to start induction radiotherapy (50 Gy in 25 fractions of 2 Gy). Despite a slight enlargement of the tumor during radiotherapy, the patient's pain was largely relieved. A cardiac magnetic resonance imaging (MRI) scan was performed, showing tumor infiltration into the pericardium and compression of the heart. However, the cardiac motion was normal, ruling out ingrowth into the myocardium. We were pessimistic about operability due to the size and the extended locoregional ingrowth of the tumor. The patient expressed a strong will to explore every possible surgical option, as this was the only curative treatment available. He was in excellent condition and had no comorbidity. We discussed the options again in our multidisciplinary chest wall expert group which comprised an oncologic surgeon, a plastic surgeon, and a thoracic surgeon; a follow-up CT scan of the abdomen and chest showed no alteration in the local situation and no signs of distant metastases. Six days later the patient was operated on. 


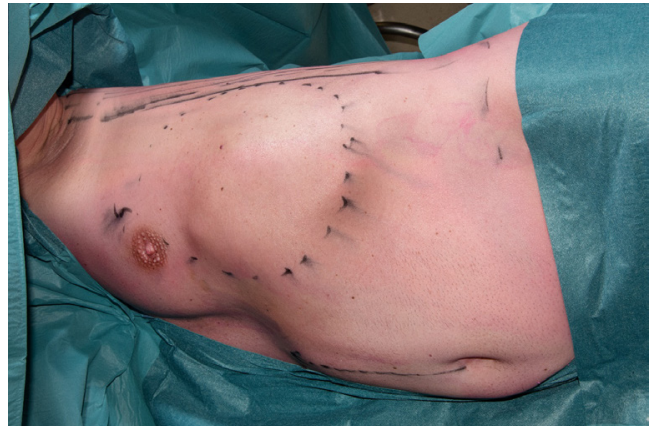

Figure 2 Patient positioned for resection with the tumor clearly visible.

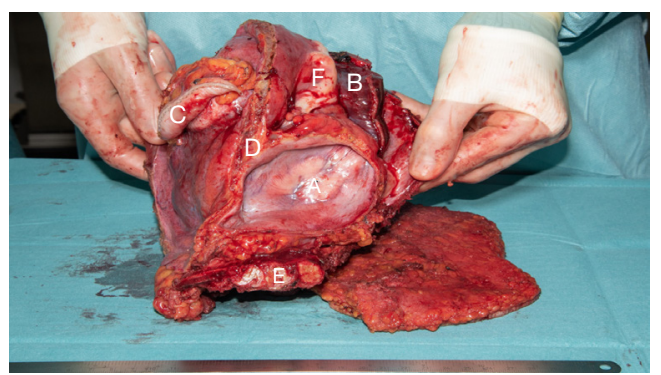

Figure 3 Resection specimen. A: inner side of pericardium with tumor ingrowth in apical region; B: wedge of left lower lobe of the lung; C: part of stomach; D: diaphragm; E: ribs; F: visible part of the tumor.

After induction of anesthesia, he was placed in a right lateral position (Figure 2). The operation started with a uniportal thoracoscopy; we removed some clear pleural fluid and did not see any pleural metastases. The left lower lobe was connected to the tumor. The tumor was attached to the pericardium which was opened away from the tumor, revealing ingrowth through the pericardium, but the heart was completely free of tumor. The tumor was also growing through the diaphragm. Upon these findings, we performed a full thickness incision around the tumor, and a skin flap was created. The vascularity of the undermined skin was compromised however, and was therefore resected together with the tumor. The pericardium was circumferentially excised away from the tumor, and a wedge resection of the left lower lobe was performed. The tumor appeared to grow into the fundus of the stomach, and this part was resected with a sufficient margin and use of a linear stapler. Finally, the involved part of the diaphragm was removed. After marking several borders of the specimen, we sent the specimen to pathology (Figure 3). On further exploration, we found a small aspecific nodule in the left triangular

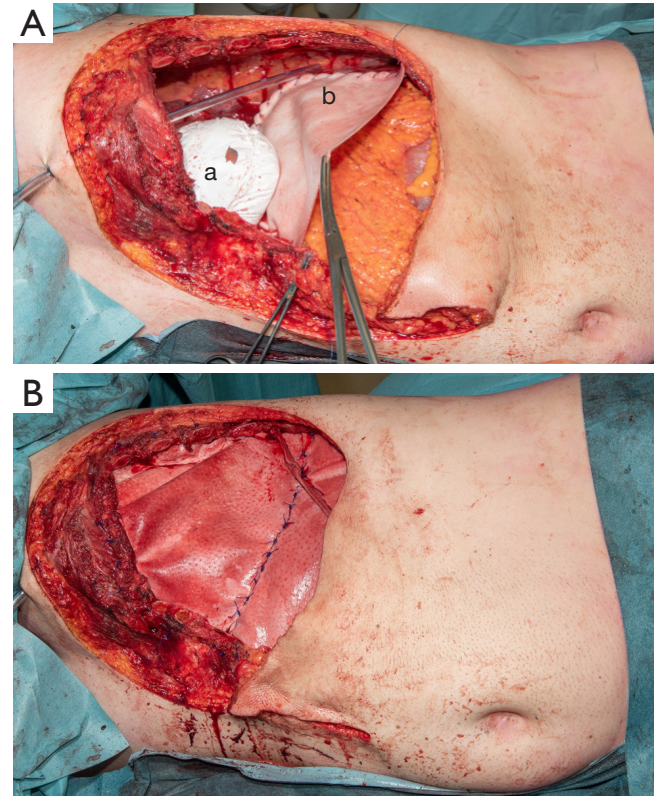

Figure 4 Patch reconstruction of pericardium and diaphragm (A) and thoraco-abdominal wall (B). (A) Reconstruction of pericardium with Gore-Tex pericardial membrane, thoracic drain in position (a), reconstruction of the diaphragm with Permacol (b). (B) Permacol implant to replace thoraco-abdominal wall. Antero-lateral border of diaphragm implant is connected to the "new" thoracoabdominal wall with a series of Prolene matrass sutures.

ligament, a few centimeters next to the tumor, which was subsequently resected.

As we needed to reconstruct a large part of the thoraco-abdominal wall including a significant part of the diaphragm, we decided to use a biological Permacol ${ }^{\mathrm{TM}}$ cross-linked collagen sheath. First, the pericardium was reconstructed using a Gore-Tex Preclude Pericardial Membrane (Gore, Dundee, UK) (Figure 4). An incision was made in the patch to allow for pericardial drainage. During the second step, a crescent-shaped Permacol implant (Medtronic, Trévoux, France) was fixed to the remaining part of the diaphragm with a Prolene $2 \times 0$ running suture. The third step included reconstruction of the thoracic wall with a large Permacol implant; the mat was first attached to the ribs with Prolene $2 \times 0$ mattress sutures, with the sutures being placed through the bone. A drain was placed in the left hemi-thorax. Next, the diaphragm patch was fixed to the large patch with Prolene $2 \times 0$ mattress sutures, completing the thoracic wall reconstruction. Finally, the inferior border of the patch was sewn to the abdominal wall (Figure 5). After completion of this part of the operation, the left latissimus 


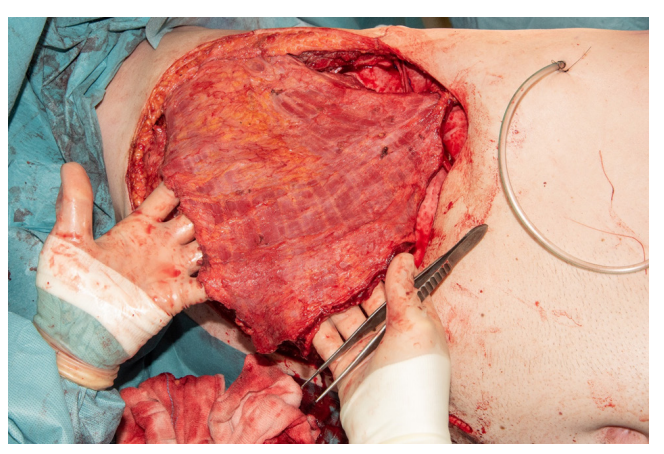

Figure 5 Latissimus dorsi muscle, ready for fixation in the defect to cover the new thoraco-abdominal wall.

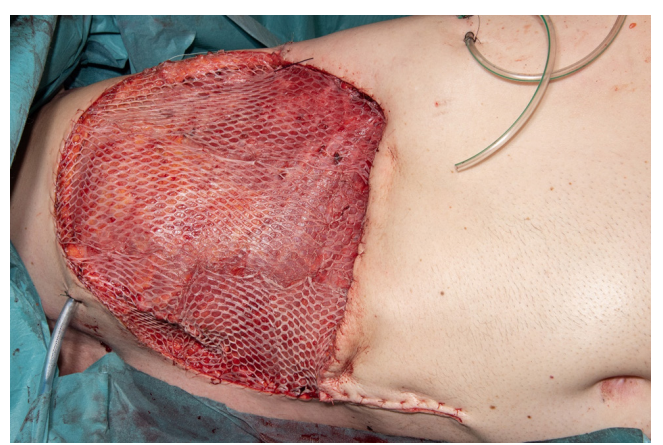

Figure 6 Meshed skin graft covering the latissimus dorsi muscle.

dorsi muscle was dissected through an incision in the skin tension lines. To prevent muscle twitching, a segment of the thoracodorsal nerve was resected. The muscle was transposed into the defect and was able to cover the whole Permacol implant (Figure 6). After placement of two drains, the muscle was attached cranially to the pectoralis muscle and caudally underneath a flap of preserved skin. Finally, the latissimus dorsi flap and pectoralis muscle were covered with a meshed split skin graft, which was harvested from the left upper leg (Figure 7).

The patient was transferred to the intensive care unit for one night. No problems with spontaneous breathing were observed. There was no moving in of the reconstruction during inspiration. The acute pain team optimized the pain medication (ketamine via epidural catheter, and intravenous fentanyl). The patient gained mobility quickly, facilitated by of his young age and good preoperative physical condition. The thoracic drain could be removed on postoperative day (POD) day 1. On POD 3 a vacuum system was placed over the split skin graft due to an abundance of serous fluid.

On POD 10, the vacuum system was removed. The

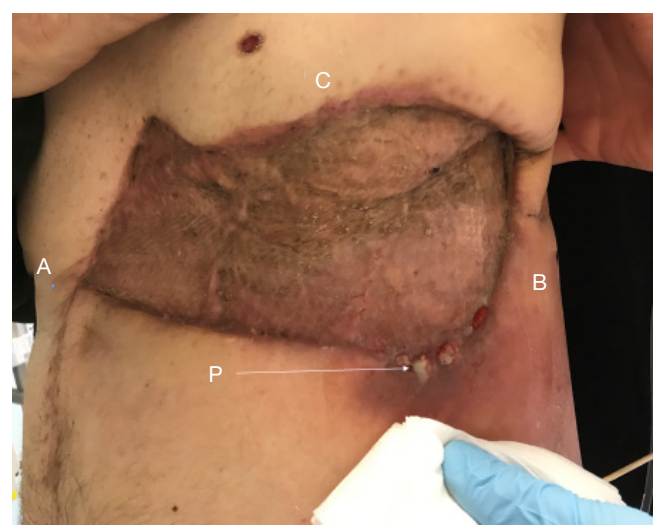

Figure 7 Situation on February 13th, 2020, patient standing. Good taking of skin graft. A: ventral; B: dorsal; C: cranial. Arrow at $\mathrm{P}$ points to small fistula with pus.

take of the skin graft was complete. Further recovery was uneventful, and the patient was discharged on POD 11.

Pathological examination revealed a grade 3 dedifferentiated chondrosarcoma, 16 centimeters in diameter. All the surgical resection margins were free of tumor. Unfortunately, the separately resected nodule in the triangular ligament of the liver appeared to be a tumor nodule, which we judged to be a peritoneal oligo metastasis. Based on the Dutch national guidelines, no adjuvant therapy was given.

On POD 45, the patient was re-admitted into our hospital because of a pneumonia of the left lower lobe and a superficial wound infection. At the lower border of the reconstruction, there was a small fistula, producing pus. Staphylococcus aureus was cultured from the pus. By exploring the fistula with a probe, we determined it to be superficial, and so we applied local flushing with $\mathrm{NaCl} 0.9 \%$ and prescribed antibiotics to the patient. Unfortunately, a CT scan showed two small tumors in the right lung which were suspect for metastases along with evidence of metastases in the left lobe of the liver. These spots were not seen on the CT scan which was taken 6 days before the resection. The patient was re-evaluated 4 weeks later. The pneumonia of his left lower lobe was resolving, the fistula produced less fluid, and there was no significant change of the two suspected lesions in the right lung (Figure 8). However, the metastases in the liver was growing, and there was a new lesion in the head of the pancreas. These findings made the prognosis very grim for the patient. Nevertheless, he was determined to start exercising in the gym again, and in March 2019, 4 months after the operation, he was able to 


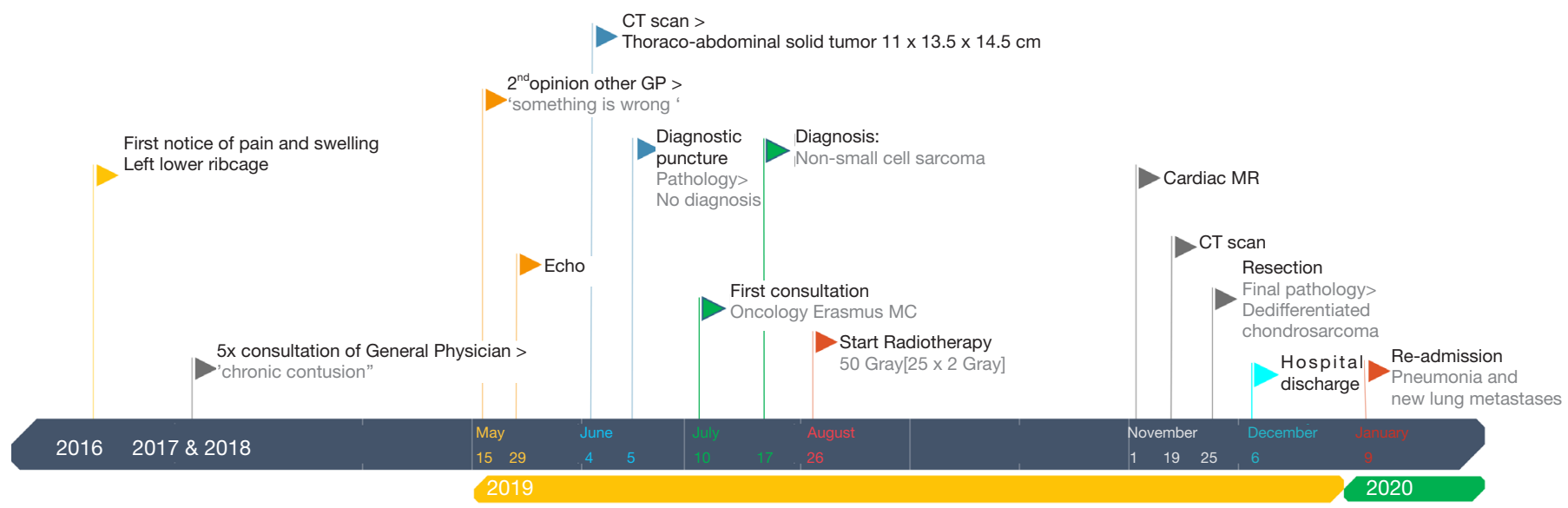

Figure 8 Timeline.

cycle 5 kilometers. Based on the above and the resolution of the infection, he received one cycle of chemo- and immunotherapy in another hospital but withdrew from further therapy due to increasing deterioration (ascites). Our patient died in May 2020. We spoke to his widow, asking what the patient's thoughts were after he realized that his prognosis was fatal. Despite the extensive surgery, the patient thought the treatment was worthwhile since he was relieved from severe and progressive pain and discomfort. The reconstruction with the latissimus dorsi flap admittedly gave him a feeling of being squeezed in the operated area, but it was not as severe as the pain he suffered pre-operatively.

All procedures performed in this study were in accordance with the ethical standards of the institutional and national research committees and with the Helsinki Declaration (as revised in 2013). Written informed consent was obtained from the patient before he deceased for publication of this manuscript and any accompanying images. A copy of the written consent is available for review by the editorial office of this journal.

\section{Discussion}

In this case, there was a very long interval between the first complaints and the final diagnosis (timeline available in Figure 8), which is not abnormal in chondrosarcoma (7). The patient himself initially attributed the pain and swelling to previous trauma, his daily physical work, and aging. $\mathrm{He}$ also felt that the GP too easily attributed the pain to being chronic contusion, and should have performed further investigations at presentation in light of persistent and worsening complaints. We question the use of chest X-ray imaging performed in January 2019, as the costosternal junction is composed of cartilage and cannot be adequately visualized on a chest $\mathrm{X}$-ray.

The question remains as to whether this tumor was a dedifferentiated chondrosarcoma from the very beginning or whether the long interval between the first complaint and final diagnosis caused this malignant dedifferentiation. There is no literature that addresses this specific question. A small series did not find a relation between delay in diagnosis and its effect on clinical outcome in high grade chondrosarcoma. The mean total delay in chondrosarcoma in this series was 688 days compared to the approximately 1,000 or more days in our patient (8). The period between May 2019-when it became clear that there was a serious problem-and the decision to operate, incurred a great deal of anxiety for the patient and his wife. The fact that the tumor did not shrink during radiotherapy led them to believe there was no hope. The decision to operate rekindled this hope, as radical surgery was the only curative treatment option.

Video-assisted thoracoscopic exploration was helpful to make the decision to proceed to an extended resection. The reconstruction performed was a two-patch technique for reconstruction of the thoraco-abdominal wall defects combined with diaphragm reconstruction and has been described by Kuwahara and colleagues (9). The choice for an acellular dermal matrix in our case proved to be the right one, given the fact that the patient developed a postoperative wound infection $(10,11)$. A few years ago, we 
used a synthetic mesh in another patient during a thoracoabdominal resection; the patient later developed a wound infection, and we had to remove the synthetic patch. In our experience with patients for whom we have performed major thoracic wall and sternum resections, there is no need to use rigid materials for thoracic wall reconstruction. Methyl methacrylate, sandwiched between two layers of mesh (9), has been associated with increased pain, excessive chest wall rigidity, infection, and fractures. Meanwhile, titanium plate fixation systems show fracture rates ranging from $0 \%$ to $11 \%$ (11).

It is unfortunate that we will never know if a shorter delay would have prevented transformation of this tumor from chondrosarcoma to dedifferentiated chondrosarcoma. A point of interest is that a CT scan made only a few days pre-operatively did rule out visible metastases while metastases became apparent on the first post-operative CT scan about 6 weeks later. Using thoracoscopy at the start ruled out inoperability during the thoracic part of the operation. We might have considered adding a laparoscopy to the initial phase of the operation but we doubt that we would have found the nodule in the ligament, and if we had recognized this metastasis, we would probably have continued with resection and reconstruction, regarding this as a single metastasis. This case does illustrate that even a very large thoracic/abdominal wall defect can be reconstructed without the use of rigid materials as proven by the fact that our patient could breathe independently immediately after the operation and could engage in athletic activity and cycling after a short period of recovery.

\section{Conclusions}

Tumors of the thoraco-abdominal wall of this size and with ingrowth in both the thoracic and abdominal structure/ organs are extremely rare. This case report, with stepby-step intra-operative photos may contribute to a better understanding of this reconstruction and help others to operate on similar patients. A progressively growing, painful tumor without evident preceding trauma should not exclusively be considered chronic contusion; proper investigation and prompt treatment are instead needed.

\section{Acknowledgments}

We would like to thank Dr. Anastasia Egorova and Dr. Edris Mahtab for their help in finalizing the manuscript and figures, and Dr. Amir Sadeghi for making the timeline.
Funding: None.

\section{Footnote}

Reporting Checklist: The authors have completed the CARE reporting checklist. Available at https://ccts.amegroups. com/article/view/10.21037/ccts-20-93/rc

Conflicts of Interest: All authors have completed the ICMJE uniform disclosure form (available at https://ccts. amegroups.com/article/view/10.21037/ccts-20-93/coif). The authors have no conflicts of interest to declare.

Ethical Statement: The authors are accountable for all aspects of the work in ensuring that questions related to the accuracy or integrity of any part of the work are appropriately investigated and resolved. All procedures performed in this study were in accordance with the ethical standards of the institutional and national research committees and with the Helsinki Declaration (as revised in 2013). Written informed consent was obtained from the patient before he deceased for publication of this manuscript and accompanying figures. A copy of the written consent is available for review by the editorial office of this journal.

Open Access Statement: This is an Open Access article distributed in accordance with the Creative Commons Attribution-NonCommercial-NoDerivs 4.0 International License (CC BY-NC-ND 4.0), which permits the noncommercial replication and distribution of the article with the strict proviso that no changes or edits are made and the original work is properly cited (including links to both the formal publication through the relevant DOI and the license). See: https://creativecommons.org/licenses/by-nc-nd/4.0/.

\section{References}

1. van Praag Veroniek VM, Rueten-Budde AJ, Ho V, et al. Incidence, outcomes and prognostic factors during 25 years of treatment of chondrosarcomas. Surg Oncol 2018;27:402-8.

2. Amer KM, Munn M, Congiusta D, et al. Survival and Prognosis of Chondrosarcoma Subtypes: SEER Database Analysis. J Orthop Res 2020;38:311-9.

3. Boehme KA, Schleicher SB, Traub F, et al. Chondrosarcoma: A Rare Misfortune in Aging Human Cartilage? The Role of Stem and Progenitor Cells in 
Proliferation, Malignant Degeneration and Therapeutic Resistance. Int J Mol Sci 2018;19:311.

4. Frassica FJ, Unni KK, Beabout JW, et al. Dedifferentiated chondrosarcoma. A report of the clinicopathological features and treatment of seventy-eight cases. J Bone Joint Surg Am 1986;68:1197-205.

5. Staals EL, Bacchini P, Bertoni F. Dedifferentiated central chondrosarcoma. Cancer 2006;106:2682-91.

6. Sakamoto A. The molecular pathogenesis of dedifferentiated chondrosarcoma. Indian J Orthop 2014;48:262-5.

7. Widhe B, Bauer HC. Diagnostic difficulties and delays with chest wall chondrosarcoma: a Swedish population based Scandinavian Sarcoma Group study of 106 patients. Acta Oncol 2011;50:435-40.

8. Goedhart LM, Gerbers JG, Ploegmakers JJ, et al. Delay

doi: $10.21037 /$ ccts-20-93

Cite this article as: Maat APWM, Zuijdendorp MM, van Leenders GJL, Hirsch A, Rothbarth J. An exceptionally large sarcoma of the thoraco-abdominal wall with ingrowth in the pericardium and stomach: a case report. Curr Chall Thorac Surg 2021;3:21. in Diagnosis and Its Effect on Clinical Outcome in Highgrade Sarcoma of Bone: A Referral Oncological Centre Study. Orthop Surg 2016;8:122-8.

9. Kuwahara H, Salo J, Tukiainen E. Diaphragm reconstruction combined with thoraco-abdominal wall reconstruction after tumor resection. J Plast Surg Hand Surg 2018;52:172-7.

10. Sodha NR, Azoury SC, Sciortino C, et al. The use of acellular dermal matrices in chest wall reconstruction. Plast Reconstr Surg 2012;130:175S-82S.

11. Sanna S, Brandolini J, Pardolesi A, et al. Materials and techniques in chest wall reconstruction: a review. J Vis Surg 2017;3:95.

(English Language Editor: J. Gray) 